\title{
Design of a novel portable fetal cardiac detection system
}

\author{
Ming Dai ${ }^{1, a^{*}}$, Xin Chen ${ }^{1}$, Kai Zhan ${ }^{1}$, Haoming Lin ${ }^{1}$, \\ Shaoxing $\mathrm{Li}^{1}$, Siping Chen ${ }^{1,2, \mathrm{~b}^{*}}$ \\ ${ }^{1}$ Shenzhen University, Shenzhen,Guangdong 518060,China; \\ ${ }^{2}$ Guangdong Key Laboratory for Biomedical Measurements and Ultrasound Imaging, \\ Shenzhen, Guangdong 518060,China \\ adaily2020@sina.com; bchensiping@szu.edu.cn
}

Keywords: Fetal cardiac monitoring; Doppler shift; Weak signal detection; Sensitivity; Noise removal

\begin{abstract}
The fetal heart rate (FHR) is a significant indicator of whether the fetus in the mother's womb is healthy. Ultrasonic Doppler detection is extensively used in the detection of FHR due to its high sensitivity, good directivity, and non-invasiveness. However, analog multiplier demodulation is usually adopted in most of the apparatuses that detect the FHR, and those devices limit their functions. Furthermore, their sensitivities are not very high. Therefore, this paper implements a new type of portable and fully digital FHR monitoring system that can be used for perinatal FHR detection. Windowed ultrasonic echo and digital multiplication methods are applied to obtain the Doppler frequency shift signal, which can be detected at specific tissue depths. Our proposed system consists of three parts: 1) the Complex Programmable Logic Device (CPLD) ultrasonic excitation and acquisition circuit, 2) the Microcontroller Unit (MCU) and peripheral circuit, and 3) the system software. Compared with two commercial fetal heart monitoring apparatuses, our results demonstrate that our proposed system can be a stable and reliable alternative for the detection of FHR.
\end{abstract}

\section{Introduction}

With higher requirements on population quality, people are paying increased attention to fetal health, and the fetal heart rate (FHR), a vital parameter, is commonly used as an indicator of maternal fetal cardiac activity and physiological health [1]. For instance, the normal fetal heart rate is about 120-160 beats/min, so when it becomes less than 99 or more than 180 beats/min, it demonstrates that the fetus has an abnormal heart rhythm [2]. FHR abnormalities often occur when the fetus is under conditions of hypoxia or ischemia or is affected by other factors, which can lead to birth defects [3]. Therefore, in order to improve the quality of childbirth, reduce infant mortality, suppress birth defects, and improve the diagnosis of mentally handicapped fetuses, we have designed a portable fetal cardiac monitoring system based on ultrasonic Doppler shift. The device is an all-digital high-precision apparatus, and can be used for perinatal Doppler FHR detection as part of homecare. The proposed system is a fast real-time fetal heart detection system that can store the FHR synchronously, and includes additional functions such as family assistance diagnosis, health guidance, health care, and remote service. As such, it has a profound practical significance in improving the population quality. 


\section{Doppler ultrasound fetal heart rate detection principle}

The Doppler effect (or Doppler shift) is the change in the frequency of a wave (or a periodic event) for an observer moving relative to the wave source [3]. When relative movement between the sound source and target occurs, the received echo frequency from the target depends on the relative speed and direction. Ultrasonic Doppler is generally used to denote the changes in the rate of fetal heart movement: as the ultrasonic probe emits an excitation signal, which is coupled into the pregnant women to be reflected by the fetal heart, the transducer receives the ultrasound echo signals. The difference between the ultrasonic transmitting and receiving frequencies allows calculation of the Doppler shift. The principle of Doppler shift allows the determination of the relationship between the moving velocity $v$ of the fetal heart and the angle $\theta$ between the direction of fetal motion and the probe.

The relationship can be written as in Eq. 1:

$$
v=\frac{f_{d} v_{0}}{2 f_{1} \cos \theta}=\frac{\left(f_{2}-f_{1}\right) v_{0}}{2 f_{1} \cos \theta}
$$

Where: $f_{1}$ is the ultrasonic excitation frequency, $f_{2}$ is the echo reception frequency, $f_{d}$ is the

Doppler shift, and $v_{0}$ is the ultrasound propagation velocity in the tissue (generally $1540 \mathrm{~m} / \mathrm{s}$ ). The

fetal heart rate can be calculated using the above formula.

\section{Fetal monitoring system design}

This portable Doppler FHR detection system consists of three parts, namely: 1) the CPLD ultrasonic excitation and acquisition circuits (with the functions of ultrasound stimulated emission and echo reception), 2) the MCU and peripheral circuits, and 3) the system software. In particular, the ultrasound stimulated emission circuit includes the functions of excitation generation, driver amplification, and impedance matching, while the echo reception circuit includes hardware filtering, echo amplification, and Analog-Digital Converter (ADC) acquisition. The CPLD generates ultrasonic excitation signals, which after a two-stage amplifying and impedance matching circuit, are transmitted to the ultrasonic probe. As the transducer receives the echo signals, these are sent to the CPLD for digital filtering and demodulation, after steps of hardware filtering, echo amplification, and ADC acquisition. In this paper, a STM32F103RCT6 is used as the control chip, and the peripheral circuit is composed of the following parts: an MCU, a LCD module, a switch button module, a Bluetooth 4.0 module, a Wi-Fi module, RS-232 serial communication modules, power modules, a SD memory module, and an audio decoding and speaker module. The overall system block diagram is shown in Figure 1.

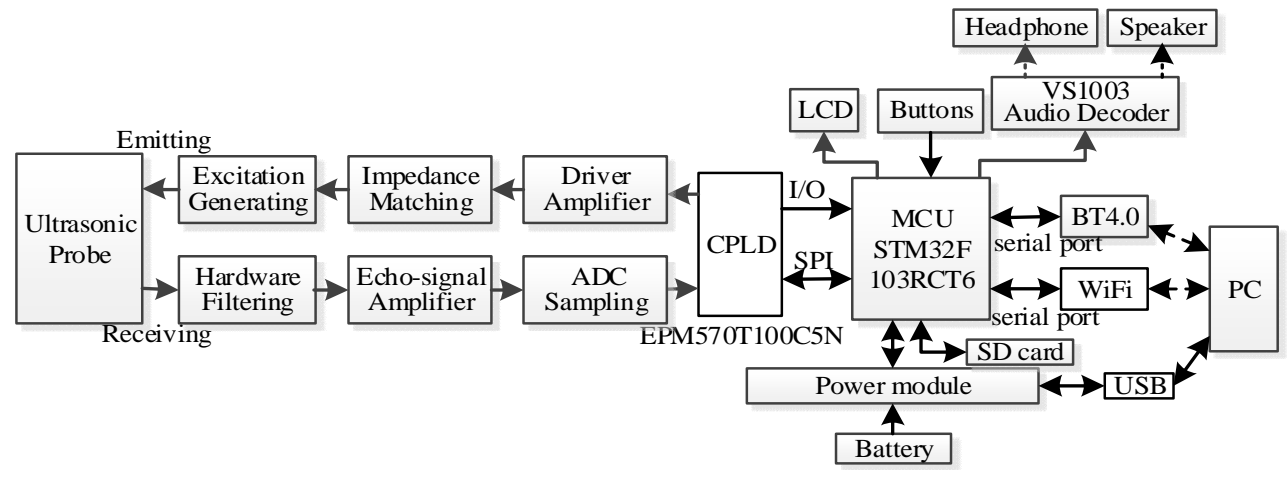

Fig. 1 Overall structure block diagram of the fetal cardiac monitoring system. 
Our proposed system uses the principle of Doppler shift to measure the FHR, and the detailed work process is as follows: the excitation signal is a square-wave pulse signal generated by the CPLD that goes through a driver amplifier and an impedance matching circuit, from which a high-frequency ultrasonic pulse sequence is generated. As the ultrasonic probe is brought close to the pregnant woman and the ultrasound pulses are properly coupled into the womb, the reflection and scattering of ultrasound waves happen once the excitation signal has encountered the fetal cardiac wall. This leads to forward or reverse Doppler frequency shift. The echo signals are detected by the ultrasonic probe, and after filtering and amplification, these signals are ADC sampled by a high-speed eight-bit chip (ADC10321), and then sent to the CPLD for further processing, such as digital filtering, multiplication, windowing, or data integration. The CPLD then calculates the Doppler shift, and finally the FHR is obtained after a significant amount of digital processing, such as finite impulse response digital filtering. The instantaneous fetal heart rate, average heart rate, probing depth, and heart rate curve are sent to the MCU via the serial peripheral interface bus for analysis, SD card recording, and displaying on an LCD. Several modes can be selected with the button module to perform the freeze, zoom, or display operations, as well as to adjust the depth of ultrasonic focus. In addition, the MCU of our proposed system is connected to Bluetooth, Wi-Fi, and USB modules through the serial port, to obtain wired or wireless data transmission using a PC. In this way, any PC equipped with the corresponding software can dynamically display in real-time the FHR and its trend curve. The audio chip (VS1003) is used for decoding the fetal heartbeat, and the fetal heartbeat sound can be heard through speakers or a headphone. In addition, the lithium battery can be charged through the USB interface and the MCU is used to manage the battery charge and discharge.

\section{Ultrasonic probe}

Since the Doppler fetal heart signal is non-stationary, the maternal breathing, heartbeat, and blood flow, as well as other factors, can easily interfere with the FHR signal. Furthermore, the fetal heart being small, it is difficult to accurately extract the weak echo signals without distortion caused by various types of interfering noise in the womb [4]. Based on the considerations above, our system uses a high sensitivity single array external transceiver, which emits at a center frequency of $1 \mathrm{MHz}$ and receives the ultrasonic echo signals. A camera picture of the transducers is shown in Fig. 2. To clearly distinguish the fetal heartbeat, several methods of increasing the ultrasonic transmitting power and improving the signal to noise ratio (SNR) are applied in order to enhance the recognition rate and meet the national standard dose of ultrasound. In this paper, the ultrasonic excitation power is less than $10 \mathrm{~mW} / \mathrm{cm}^{2}$.

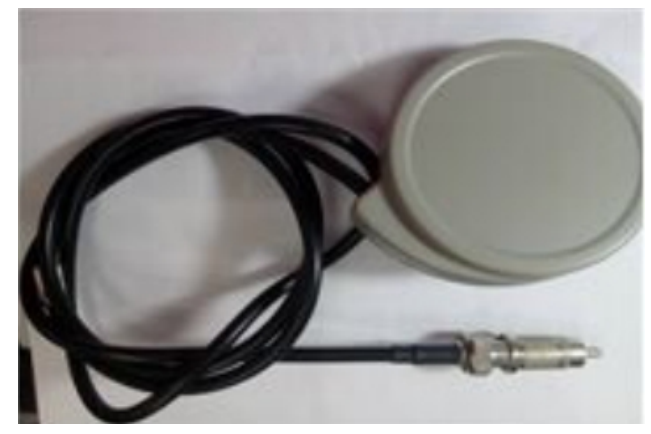

Fig. 2 Camera picture of the ultrasonic transducers.

\section{Ultrasonic excitation circuit}

The ultrasonic excitation circuit consists of three parts: 1) an excitation generator circuit, 2) an impedance matching circuit, and 3) a driver amplifying circuit. The ultrasonic excitation and amplifying circuit are shown in Fig. 3. The ultrasound array element inside the probe is a capacitive load; thus, to match the capacitive load with the driving circuit and ensure that the ultrasonic excitation signal is without significant distortion, the capacitive load part should have no effect on the 
diagnostic excitation signal. In the ultrasonic excitation circuit, transformer coupling is employed to realize impedance matching and ultrasonic excitation circuit is imposed on the two ends of the probe array element. In addition, this ultrasonic high-frequency excitation source is generated by a CPLD (EPM570T100C5N) made by Altera in USA, which is an ultrasonic excitation control core unit. The CPLD generates a high-frequency pulse excitation signal source through Verilog language programming, and then an EL7222 is used to amplify the high-frequency pulse excitation signal. A transformer $\mathrm{T} 1$ is used as an insulator between the excitation circuit and ultrasonic probe.

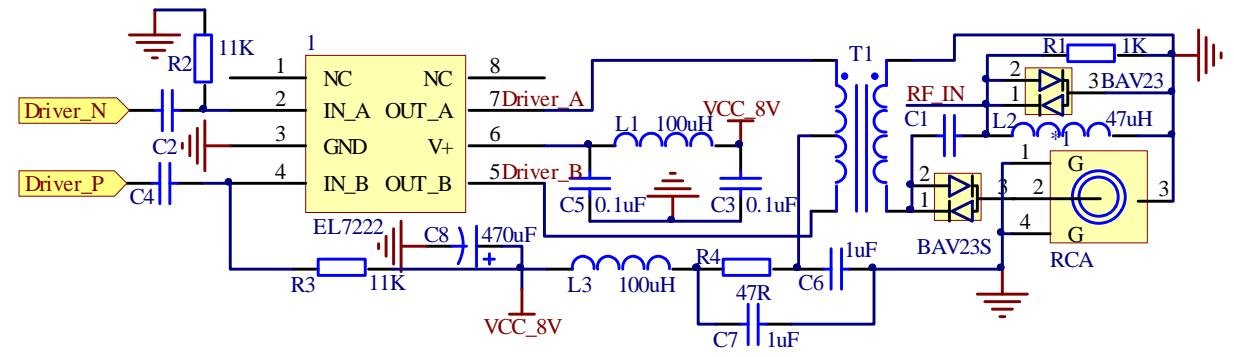

Fig. 3 Electric diagram of the ultrasonic excitation circuit.

\section{Ultrasonic echo detection circuit}

As the ultrasonic echo signals are weak [6], it is difficult to distinguish multiple invalid echo signals from various interferences. Therefore, in order to improve the SNR of echo signals, an OPA2356 is used as a band-pass filter (the fetal Doppler shift frequency generally varies from $50 \mathrm{~Hz}$ to $200 \mathrm{~Hz}$ [5]) to reduce the influence of interfering signals, and an amplifier is used to pre-amplify the valid echo signal and ADC10321 is used to ADC sample the signal in this work. Afterwards, the ultrasonic echo signal is sent to the CPLD for digital filtering, windowing processing, digital multiplying, and data integration. Finally, the CPLD calculates the value of Doppler shift and the FHR is obtained. The electric diagram of the ultrasonic echo detection circuit is presented in Fig. 4.

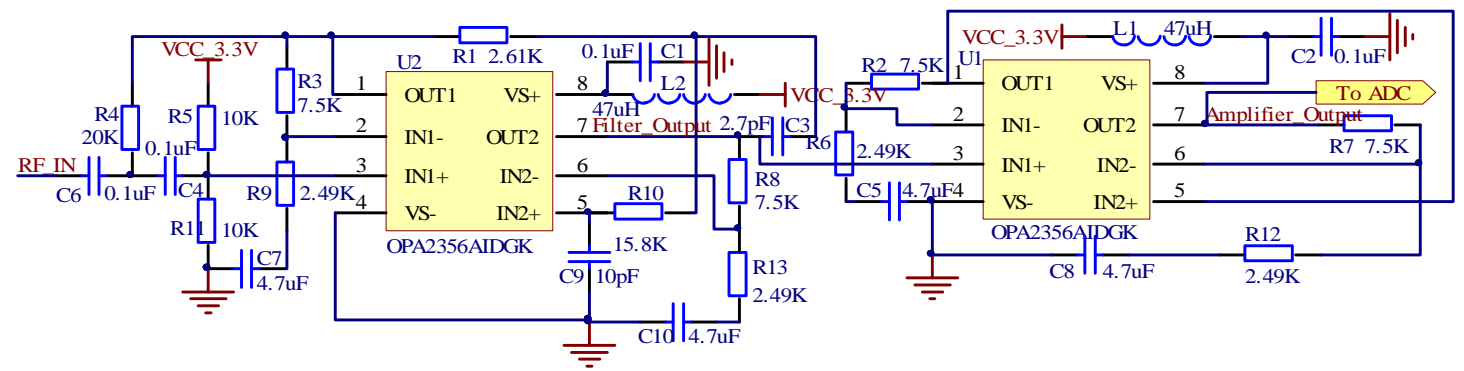

Fig. 4 Electric diagram of the ultrasonic echo detection circuit.

\section{Power module}

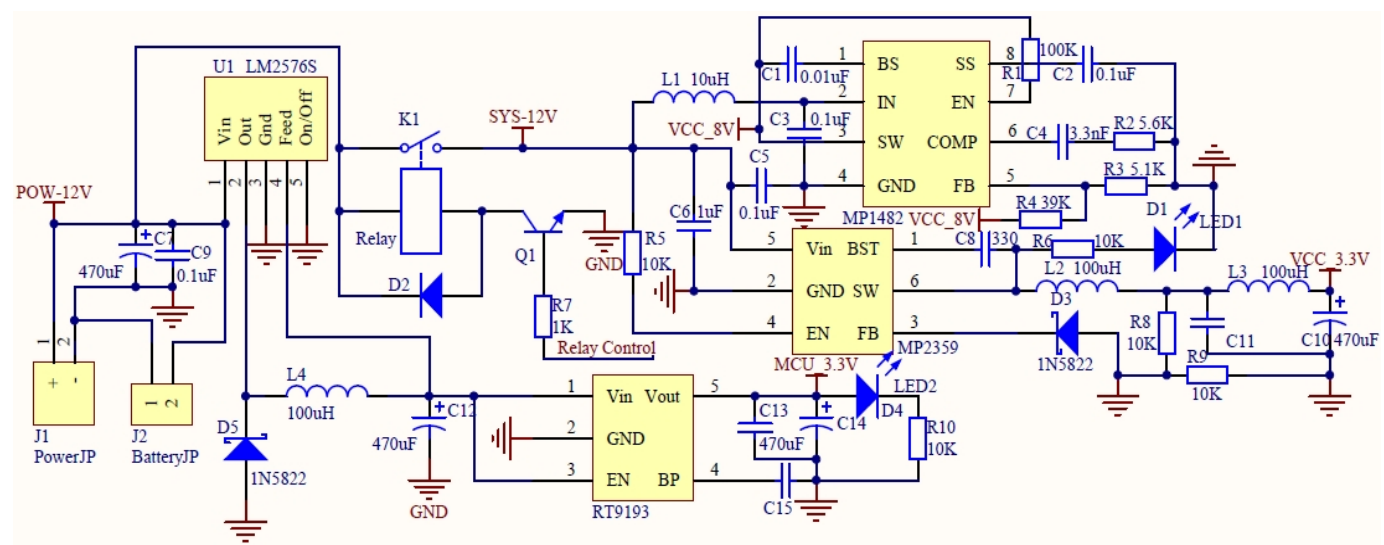

Fig. 5 Circuit diagram of the power module of this proposed system. 
The system is powered by a single $12 \mathrm{~V}$ DC power supply. The $12 \mathrm{~V}$ voltage is transformed into 5V by a LM2576 (a DC-DC converter), which is in turn transformed into 3.3V by a RT9193 to supply power for the MCU. Additionally, the MP1482 is used to generate $8 \mathrm{~V}$ to supply the ultrasonic driver chip (EL7222). Our proposed system starts in standby mode after the power supply is switched on, and the MCU enters the normal operating mode when the boot button is pressed. Whether the relay is open or closed is decided by Q1, which is controlled by the MCU. When the relay is closed, a 3.3V voltage is generated by MP2359, which supplies peripheral circuits. To reduce the system power consumption as much as possible, we implement the following procedure when ultrasonic echo signals cannot be detected by the ultrasound probe within $50 \mathrm{~s}$ : the MCU closes the relay to cut off the power supply for the peripheral circuits and the ultrasonic driver circuit, until the measure button is re-pressed, which restarts the relay $\mathrm{K} 1$ in order to supply power. The power module circuit diagram is presented in Fig. 5.

\section{System software design}

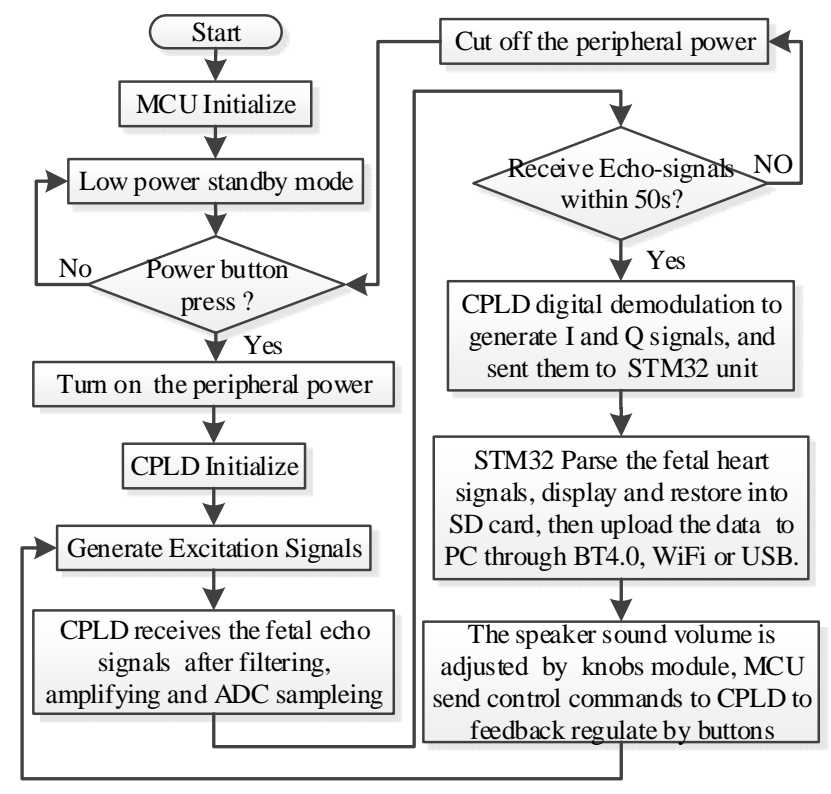

Fig. 6 Software flow chart of the proposed system.

Our proposed FHR detecting system adopts several ways to reduce the system power consumption. Before starting, the MCU is in low power standby mode, while power supply to the peripheral circuits and ultrasonic excitation circuit is cut off. As the system is in normal operation mode, if the ultrasound probe does not receive valid echo signals within $50 \mathrm{~s}$, the MCU controls the relay $\mathrm{K} 1$ to shut down power to the peripheral circuits and ultrasonic driver circuit. Meanwhile, the system enters a low-power standby mode until it is restarted by pressing the power button. When the system reboots, the MCU starts initializing, then switches on the relay K1 to supply power to the peripheral and ultrasonic driver circuits, after which an initialization procedure takes place in the CPLD. Under control of the MCU, the ultrasonic excitation pulse signals with $1 \mathrm{MHz}$ center-frequency are generated though the CPLD. These signals are then amplified by the driver amplifier circuit and transmitted to the ultrasonic probe after impedance matching. Furthermore, the probe also receives the ultrasonic echo signals, which are sent to the CPLD for further processing after hardware filtering, amplification, and ADC acquisition. After the CPLD receives the FHR digital echo signal, the first echo signals will be generated after depth selection of ultrasonic detection and will be windowed though a windowing function sequence. Consequently, two preset echo signals are produced: signal $\mathrm{I}$ is obtained by integrating the product of one preset echo signal with its cosine 
expansion series, while signal Q is obtained by integrating the product of the other preset echo signal with its sine expansion series. The CPLD then sends the two signals to the MCU for display, and the heartbeat rate can be obtained as an output via VS1003. Meanwhile, as the MCU receives the I and Q signals, it sends control commands to the CPLD to feedback-control the echo excitation output module. In addition, the output of the speaker volume can be adjusted by a knob controlled by STM32. The operation instruction of the button can be recognized by MCU using the external interrupt control method. Finally, the detection depth and ultrasonic power can be adjusted by the CPLD. The software flow chart is shown in Figure 6.

\section{System functionality testing}

Table 1 Comparison data and accuracy analysis of our proposed FHR detection system

\begin{tabular}{lllllc}
\hline \multicolumn{2}{c}{ Testing No. } & ALK(Sweden) PS-320 & \multicolumn{4}{c}{ different fetal simulator signals (per min) } \\
\hline PS-320 Output & 60 & 90 & 120 & 150 & 180 \\
Sonoline B (per min) & 59 & 90 & 119 & 152 & 177 \\
Accuracy -Sonoline B (\%) & $98.3 \%$ & $100 \%$ & $99.2 \%$ & $98.6 \%$ & $98.3 \%$ \\
H3-T (per min) & 62 & 88 & 123 & 147 & 181 \\
Accuracy -H3-T (\%) & $96.7 \%$ & $97.8 \%$ & $97.5 \%$ & $98.0 \%$ & $99.4 \%$ \\
Our system (per min) & 58 & 89 & 121 & 148 & 177 \\
Accuracy of this system(\%) & $96.7 \%$ & $98.8 \%$ & $99.1 \%$ & $98.7 \%$ & $98.3 \%$ \\
\hline Average accuracy of this system (\%) & \multicolumn{5}{c}{$98.32 \%$} \\
\hline
\end{tabular}

To test the accuracy and reliability of this portable ultrasonic FHR detection system, two commercially available high-precision portable fetal heart detectors were chosen for comparison. In our experiments, the PS-320 produced by the ALK company in Sweden was used to generate 90, 120, 150, 180 beats/min fetal simulator signals. Our proposed FHR detecting system, the Sonoline-B designed by Kangtai in China, and the H3-T manufactured by Beibinfeng in China are used to measure those five fetal simulator signals for $10 \mathrm{~min}$. Table 1 shows the averaged FHR values recorded during that period, along with the data for 15 experiments, with accuracy analysis of the three apparatuses.
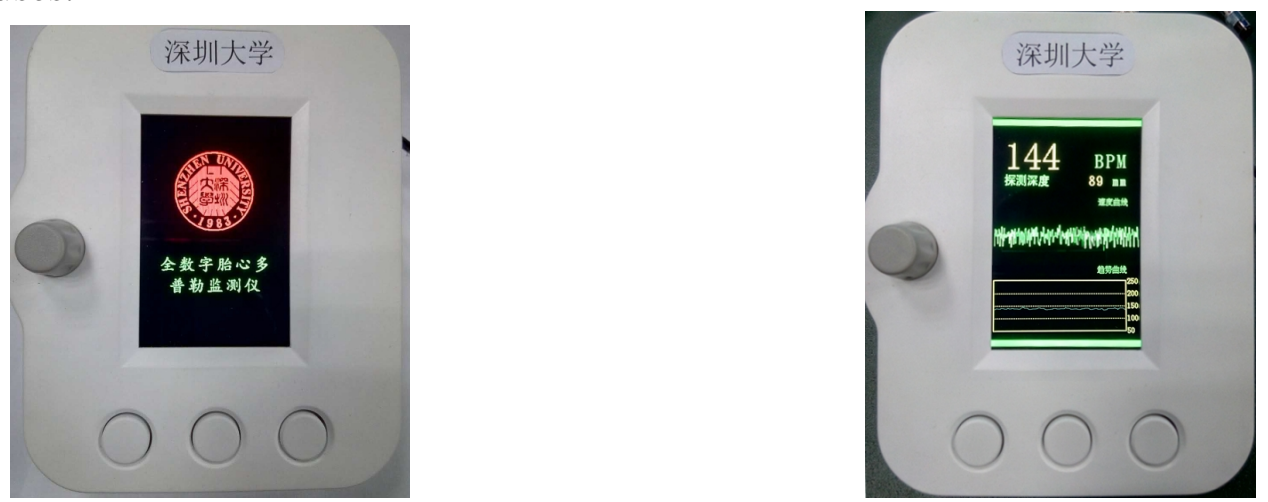

Fig. 7(a) Splash screen of this proposed system; Fig. 7(b) Screenshot of practicality display.

As shown in Table 1, our portable FHR detecting system was used to measure the five fetal simulator signals and the results demonstrate an accuracy consistently greater than $96 \%$, with an average value of $98.32 \%$. This value is comparable to those of the two other FHR measuring devices, the Sonoline-B (98.88\%) and the H3-T (97.88\%). This illustrates that our proposed system is not significantly different from the two commercially available apparatuses, and indicates that our portable ultrasonic Doppler fetal heart rate detection system has the advantage of high accuracy. Therefore, our proposed portable FHR detection system can be widely applied for fetal cardiac health 
monitoring for improvement of population quality.

In addition, in order to further test the functionality of the system, we conducted an experiment on a pregnant woman with an 18 week old fetus. Fig. 7 (a) shows the splash screen and Fig. 7 (b) shows a screenshot of the practicality appearing during the experiment. In the latter, the velocity curve of the Doppler shift signal has a high SNR, and the output amplitude as well as the frequency range of FHR meet the design requirements. The current detection depth, the Doppler shift velocity curve, and the average FHR can also be displayed in real-time on the LCD.

\section{Conclusions}

This paper introduces a portable ultrasonic Doppler fetal heart rate monitor, and the prototype system is described. Currently, the traditional analog multiplier method is the most common way to obtain fetal ultrasound frequency shift information. We adopted a different method, in which the ultrasonic echo signals are multiplied to their sine and cosine expansion series to obtain the Doppler shift information and finally the fetal heart signal. Compared with other ultrasonic testing instruments sold on the market, our system not only has the option of detection distance selection, but can also detect the specific depth of the tissue. Moreover, this system not only allows the selection of focal depth through the button control module and can display the FHR and current detection depth in real-time, but can also dynamically display the fetal heart rate curve and the FHR trend curve. In addition, the data can be stored on an SD card and be uploaded to a PC for further processing. Compared with the two other commercially available apparatuses, the experimental results show that our proposed FHR monitoring system can be applied for continuous and real-time measurement of the FHR. Besides, the system power consumption is low, and there is no significant difference in accuracy between our system and the two commercially available apparatuses. Our system can detect the specific depth and record the cardiac health information of the fetus. Finally, the modular design is introduced in this FHR detection system. In summary, our system has the advantages of simple operation and adjustable detection depth, and can be used by pregnant women at home. It offers a portable and accurate FHR monitoring, with a great application potential that can significantly improve the quality of childbirth, reduce the fetal death rate or the birth of mentally handicapped children and suppress birth defects.

\section{Acknowledgment}

This work was supported by the National Natural Science Foundation of China (Grant NO. 61372006 and NO. 61427806).

\section{References}

[1] Sampson M B, Mudaliar N A, Lele A S, Fetal heart rate variability as an indicator of fetal status J. Sci. Postgraduate medicine, 1980, 67(5): 207-10, 213-5.

[2] Information on http://radiopaedia.org/articles/fetal-heart-rate

[3] Information on https://en.wikipedia.org/wiki/Doppler_effect

[4] Zhang C, Zhang S, Gao S, An improved auto-correlation method for Doppler fetal heart rate measurement, J. Sci. Journal of Biomedical Engineering, 2001, 18(3): 434.

[5] Liang W, Que P, Optimal scale wavelet transform for the identification of weak ultrasonic signals, J. Sci. Measurement, 2009, 42(1): 164-169. 